\title{
Ethics and Professionalism in Medical Education
}

\author{
Karunathilake, I.M.
}

\section{Introduction}

The complex and unique interactions in Medical Education are intricately linked to ethics, moral values and professionalism. These complex interactions occur between medical teachers, students, patients and their families, academic institutions, statutory bodies, cooperate sector and the community.

\section{Different Roles of a Medical Teacher and Their Ethical Implications}

The range of ethical and moral responsibilities of a medical teacher can be explained by considering the different roles of a teacher. i.e. information provider, role model, facilitator, assessor, planner and resource developer.

As information providers, medical teachers have the responsibility of providing knowledge that is relevant, accurate and up-to-date. As a role model, the teacher must respect the confidentiality and safeguards the privacy of learners and patients. Any use of a patient for teaching purposes should take place only with the consent of that patient or guardian. Where the contact between the learner and the patient is primarily for teaching purposes, then explicit prior consent must be obtained from the patient.

No longer is the teacher seen predominantly as a dispenser of information. The principles of adult learning require a teacher to be a facilitator or manager of the trainee's learning. Mentoring is an important responsibility as a facilitator of learning.

Professor and Head,

Department of Medical Education,

Head, WHO Collaborating Centre for Medical Education, Faculty of Medicine, University of Colombo, Sri Lanka.

Email: karunathilake@hotmail.com
It is a process whereby an experienced, highly regarded, empathic person (the mentor), guides another individual (the mentee). It is a two way relationship between the mentor and the mentee. The mentor has interest in the professional and personal development of the mentee and should maintain a professional relationship, avoiding discrimination, inappropriate relationships or harassment. As a supervisor, medical teacher should provide guidance and feedback and adhere to research ethics.

The role of student assessor is important and challenging with profound legal and ethical issues. The examiner should treat all candidates with respect regardless of level of training, race, creed, colour, gender, sexual orientation or field of study and ensure that each candidate is treated equitably. The examiners must adhere to the laid down regulations and pay heed to recognized norms of assessment. The candidates' marks grades or performance should not be divulged by the examiner to unauthorized individuals. The examiner should not attempt to influence the assessment of a candidate by another examiner or fraudulently alter the marks or grades of any candidate. Any conflict of interest that is likely to or would be perceived to be affecting an independent assessment of any candidate needs to be declared.

\section{Interactions with the Community}

Teachers will keep in mind that, in the field of Medicine, the knowledge and skills which they share with learners and their peers have been born out of human suffering. Public and private education and the prevailing health system in the country should be protected by the teachers. High quality education is the common goal of the public, education institutions and teachers and a cooperative effort is essential among these groups to attain that goal. Medical Education needs to understand and respect the values and 
traditions of the diverse cultures represented in the community. Curriculum planning should be based on heath needs of the community. This presents a significant challenge for the teacher.

\section{Moral and Ethical Responsibilities of Learners}

Moral and Ethical Responsibilities of students include pursuing excellence in their acquisition of knowledge, skills and attitudes in the medical profession. They must behave with honesty, integrity and fairness in all their dealings and respect the dignity of patients. It is unethical and unprofessional for students to engage in academic or research misconduct including misrepresentation, plagiarism or fraud.

\section{IT in Medical Education and Ethics}

The expanding role of IT in Medical Education will have both positive and negative implications on ethics and professionalism.
The next generation of medical students will be digital natives as opposed to the medical teachers who are digital settlers or digital immigrants. Guidelines need to be in place to ensure that the full potential of IT is available for medical education and to avoid possible adverse impacts.

\section{Conclusion}

Medical Education, due to its unique complex interplay between different stakeholders, is intricately linked with ethics and professionalism. These factors need to be considered when planning Medical Education for the future.

\section{Reference}

Crosby, R.H.J. (2000) AMEE Guide No 20: The good teacher is more than a lecturer-the twelve roles of the teacher, Medical teacher, 22, 4, pp. 334-347. 